\title{
Solving Kuramoto-Sivashinsky equation by the new iterative method and estimate the optimal parameters by using PSO algorithm
}

\author{
Karam Adel Abed \\ Department operations Research and intelligence techniques, College of Computer Sciences and Mathematics, \\ University of Mosul, Iraq
}

\begin{tabular}{l} 
Article Info \\
\hline Article history: \\
Received Dec 21, 2019 \\
Revised Feb 28, 2020 \\
Accepted Mar 13, 2020 \\
\hline
\end{tabular}

Keywords:

New iterative method Partial differential equation PSO algorithm

\begin{abstract}
In this study, optimal parameters estimation is performed for non-linear (Kuramoto-Sivashinsky) equation using a crossbred method between Particle swarm optimization (PSO) algorithm and (NIM) technique and for the appropriateness we call it (PSO-NIM). It turns out that the optimal parameters significantly improve the solutions when we use a fitness function suitable for the issue. The results confirmed that the get approximate solutions are in suitable pact with the exact solutions and the proposed method provides high accuracy and efficiency in comparison with (NIM) which use traditionally chosen parameters.
\end{abstract}

\section{Corresponding Author:}

Karam Adel Abed,

Department operations Research and intelligence techniques,

College of Computer Sciences and Mathematics,

University of Mosul, Mosul, Iraq.

Email: karamadel@uomosul.edu.iq

\section{INTRODUCTION}

Most physical phenomena can generally be described as partial differential equations, whether in airflow on aircraft objects, spacecraft movement, underwater sound signal processing, crystal growth, population growth rate, e-marketing, stock exchange or nuclear explosions, in fact, most mathematical physics is partial nonlinear differential equations. Although abstraction converts the equations under study into ordinary differential equations, the full description of these systems falls within the general domain of partial differential equations $[1,2]$.

One of the most recent ways to solve nonlinear partial differential equations is new iterative method, the first to introduce this method is Daftardar-Gejji and Jafari in 2011 [3]. and modified by Hameda [4, 5].

The (NIM) technique is one of the most modern methods at present. It is a valuable tool for specialized researchers in applied mathematics because it provides immediate symbolic limits for analytical solutions instead of numerical approximation of all solutions for linear and nonlinear partial differential equations.

The new iterative method has good results and is easy to execute utilize the computer and was proved successful after it was applied to a different set of differential equations of the right rank and fractional [6-11]

The Kuramoto-Sivashinsky equation is a pattern of a nonlinear PDE that we see much in the study of continuous media that exhibit messy conduct, the partial and normal differential equations of fractional and correct order, integrative equations, integral differential equations are applications to a set of linear and nonlinear equations [12]. Particle swarm optimization (PSO) algorithm is proposed by Eberhart and Kennedy (1995) which simulates social behavior including fish, birds, etc. [13], The Particle Sprinkler Optimization 
(PSO) algorithm is a common method and has many known uses. It is one of the most effective mathematical methods in selecting the best parameter values. If compared to genetic algorithms, it is ideal efficiency in accurate calculations and is easy to implement. The Particle swarm optimization (PSO) algorithm is development for genetic algorithms, where crossover, mutation was canceled and is therefore widely used to solve scientific problems and is used to study and apply partial differential equations [14]. The Figure 1 illustrates the basic idea that starts from random solution to optimal solution after continuous repetition and evaluation fitness [14]:

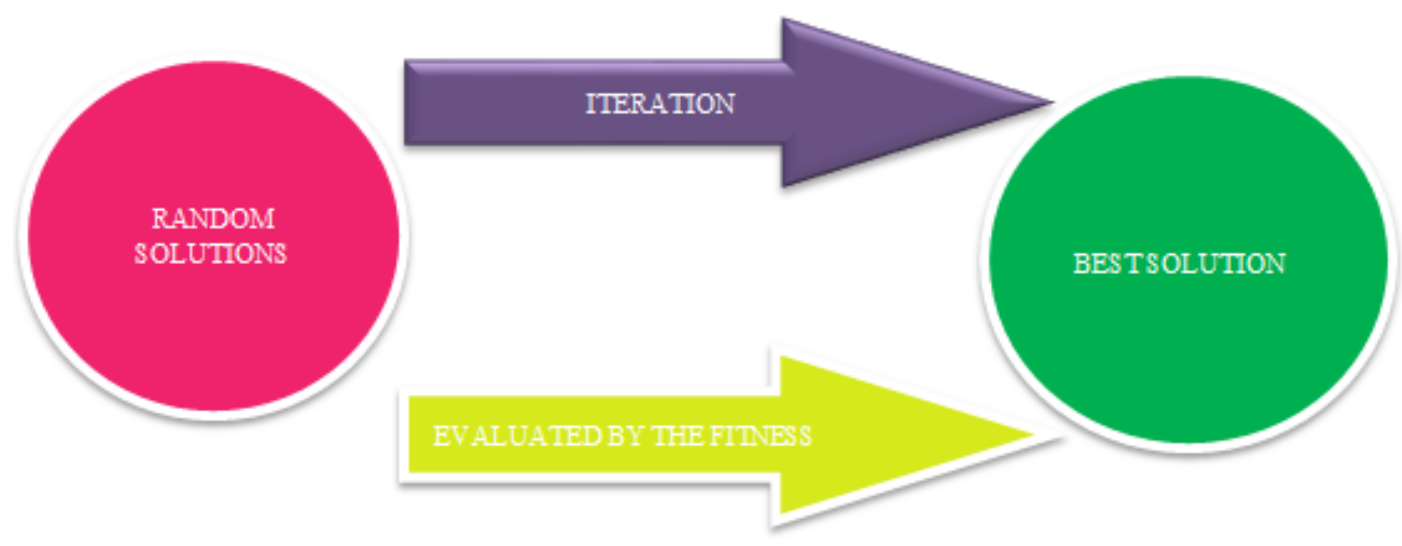

Figure 1. PSO algorithm for solution the optimal

\section{MATHEMATICAL MODEL:}

This equation (KS) was derived through Sivashinsky as a paradigm for plane flame spread [15], and by Kuramoto as a pattern for phase disorder in reaction propagation systems [16, 17].The KuramotoSivashinsky equation has many applications in various physical, scientific and engineering phenomena, such as reaction diffusion systems, flow of thin liquid membranes [18] and in the model of diffusion and chaos [19-22]. Many authors have studied Kuramoto-Sivashinsky equation numerically in several different methods Homotopy Perturbation Method, Adomian Decomposition Method, Differential Transform Method [23, 24]. Consider the Kuramoto-Sivashinsky equation with [25]:

Consider the Kuramoto-Sivashinsky equation with [27]:

$\frac{\partial Y}{\partial t}+u \frac{\partial Y}{\partial x}+\alpha \frac{\partial^{2} Y}{\partial x^{2}}+\gamma \frac{\partial^{4} Y}{\partial x^{4}}=0, t>0$

The initial condition and boundary condition are taken away from the exact solution [26-28]:

$$
Y(x, t)=\beta+\frac{15}{19} * \sqrt{\frac{11}{19}}\left(-9 \tanh (k(x-\beta t-r))+11 \tanh ^{3}(k(x-\beta t-r))\right)
$$

To illustrate the main idea of the (NEM) method by taking the next differential equation:

$$
B=g+A(B)
$$

Where $\mathrm{M}$ is a nonlinear term, we are assuming that $\mathrm{M}$ in (1) represent the series form:

$$
A\left(\sum_{i=0}^{\infty} B_{i}\right)=A\left(B_{o}\right)+\sum_{i=1}^{\infty}\left[A\left(\sum_{j=0}^{i} B_{j}\right)-A\left(\sum_{j=0}^{i-1} B_{j}\right)\right]
$$

Can be decayed decomposed the nonlinear operator $\mathrm{M}$ as:

$$
\sum_{i=0}^{\infty} B_{i}=g+A\left(B_{o}\right)+\sum_{i=1}^{\infty}\left[A\left(\sum_{j=0}^{i} B_{j}\right)-A\left(\sum_{j=0}^{i-1} B_{j}\right)\right]
$$

By compensation (4) and (5) in (3), get on: 


$$
\left.\begin{array}{c}
B_{1}(x, t)=A\left(B_{o}(x, t)\right) \\
\left\{\begin{array}{c}
B_{2}(x, t)=A\left(B_{o}(x, t)+B_{1}(x, t)\right)-A\left(B_{0}(x, t)\right) \\
B_{3}(x, t)=A\left(B_{o}(x, t)+B_{1}(x, t)+B_{2}(x, t)\right)-A\left(B_{o}(x, t)+B_{1}(x, t)\right. \\
\vdots \\
\vdots
\end{array}\right\} \\
B_{i+1}=\left(A\left(B_{o}(x, t)+B_{1}(x, t)+\cdots+B_{n}(x, t)\right)-\left(A\left(B_{o}(x, t)+B_{1}(x, t)+\cdots+B_{n-1}(x, t)\right)\right.\right. \\
; n=1,2,3,4, \cdots \cdots \cdots \cdots \cdots
\end{array}\right\}
$$

Where:

To understand the duplicate relation in the following manner:

$$
\sum_{i=0}^{\infty} B_{i}=g+A\left(\sum_{j=0}^{\infty} B_{j}\right)
$$

The $\mathrm{n}$-borders solutions of (4) is given by $\approx B_{o}+B_{1}+B_{2}+\cdots \cdots+B_{n-1}$, the convergence of this technique in [29-31].

\section{APPLICATION}

In this part, we enforcement to solve $B(\mathrm{x}, \mathrm{t})$ the initial conditions which satisfying $(1)$ :

$Y(h, 0)=\beta+\frac{15}{19} * \sqrt{\frac{11}{19}}\left[-9 \tanh (k(x-\beta t-r))+11 \tanh ^{3}(k(x-\beta t-r))\right]$

Now, by the integral for the (1), we get:

$B(x, t)=\int_{0}^{t} A(B(h, t)) d t=\int_{0}^{t}\left(Y \frac{\partial B}{\partial h}+\alpha \frac{\partial^{2} B}{\partial h^{2}}+\gamma \frac{\partial^{4} B}{\partial h^{4}}\right) d t$

With the initial conditions in (10) we get:

$B_{1}(h, t)=\int_{0}^{t} A\left(B_{o}\right) d t=\int_{0}^{t}\left(B_{o} \frac{\partial B_{o}}{\partial h}+\alpha \frac{\partial^{2} B_{o}}{\partial h^{2}}+\gamma \frac{\partial^{4} B_{o}}{\partial h^{4}}\right) d t$

After solving (11) we get:

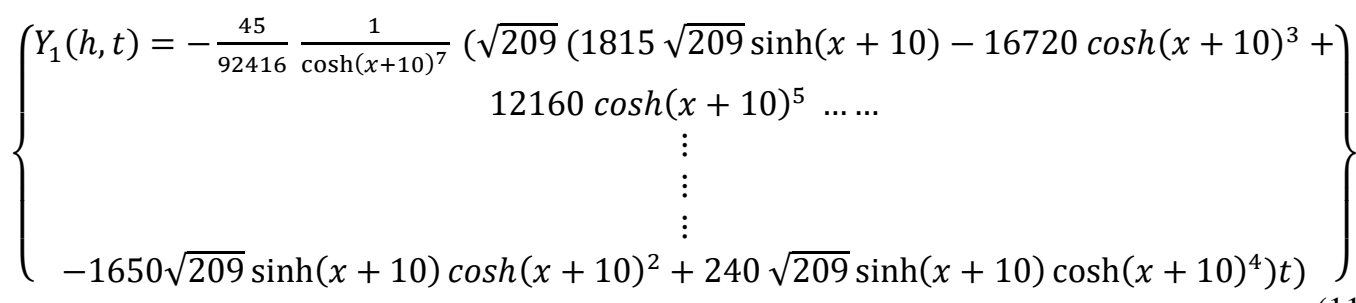

Now, we work to find the border second:

$B_{2}(h, t)=\int_{0}^{t} A\left(B_{o}(h, t)+B_{1}(h, t)\right)-A\left(B_{0}(h, t)\right) d t$

Then

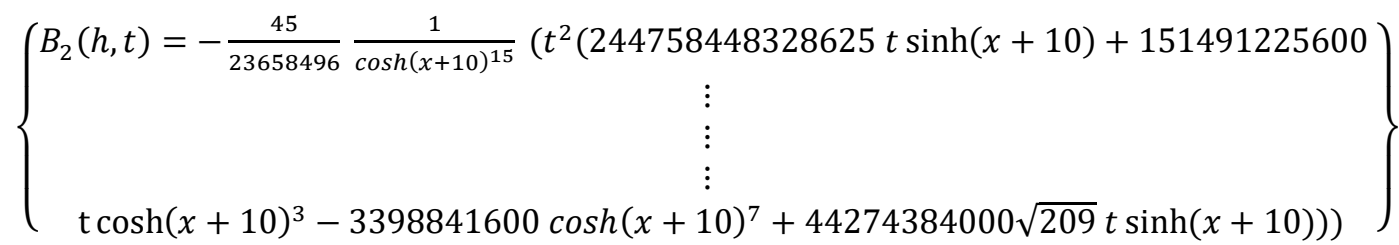


And the finally we find $\mathrm{Y}_{3}$ :

$$
\left\{\begin{array}{c}
B_{3}(h, t)=-\frac{15}{39180} \frac{1}{\cosh (x+10)^{31}}\left(t ^ { 3 } \left(-554701 \cosh (x+10)^{23}+484861 \cosh (x+10)^{29}\right.\right. \\
\vdots \\
\vdots \\
\vdots \\
\ldots \ldots \ldots \ldots \ldots+26471 \cosh (x+10)^{12} t \sqrt{209} \sinh (x+10)-39803 \cosh (x+10)^{19}
\end{array}\right\}
$$

It was found that the error in the numerical method reached 10-5 at its lowest level and 10-9 at its best, as shown in Table 1 and Figures 2 and 3.

Table 1. Absolute error of the $\left|B_{\text {exact }}-B_{N I M}\right|$ by using parameters $r=-12, \beta=5, k=\frac{1}{2} \sqrt{\frac{11}{19}}, \alpha=1$, with $3^{\text {nd }}$ order at $\mathrm{t}=0.1$

\begin{tabular}{lccccc}
\hline \multicolumn{5}{c}{$\mathrm{T}$} \\
\hline $\mathrm{H}$ & 0.1 & 0.2 & 0.3 & 0.4 & 0.5 \\
8 & $4.564000 * 10^{-6}$ & $1.409900 * 10^{-5}$ & $3.059800 * 10^{-5}$ & $5.564700 * 10^{-5}$ & $9.028500^{-5} 10^{-5}$ \\
15 & $2.000000 * 10^{-8}$ & $6.700000 * 10^{-8}$ & $1.470000 * 10^{-7}$ & $2.740000 * 10^{-7}$ & $4.420000 * 10^{-7}$ \\
17 & $3.000000 * 10^{-9}$ & $1.400000 * 10^{-8}$ & $3.700000 * 10^{-8}$ & $5.900000 * 10^{-8}$ & $9.600000^{-8} 10^{-8}$ \\
20 & $1.000000 * 10^{-9}$ & $2.000000 * 10^{-9}$ & 0 & $2.000000 * 10^{-9}$ & $1.000000^{-9} 10^{-8}$ \\
30 & $1.000000 * 10^{-9}$ & $1.000000 * 10^{-9}$ & $1.000000 * 10^{-9}$ & $1.000000 * 10^{-9}$ & $1.000000 * 10^{-9}$ \\
\hline
\end{tabular}

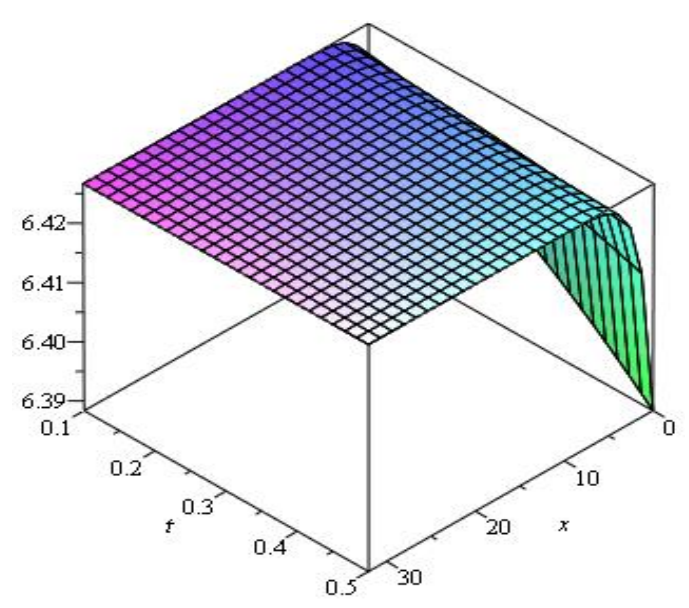

Figure 2. The function $(B)$ for the two borders approximation $(B-2)$ by using NIM, when $\mathrm{t}=0.1$

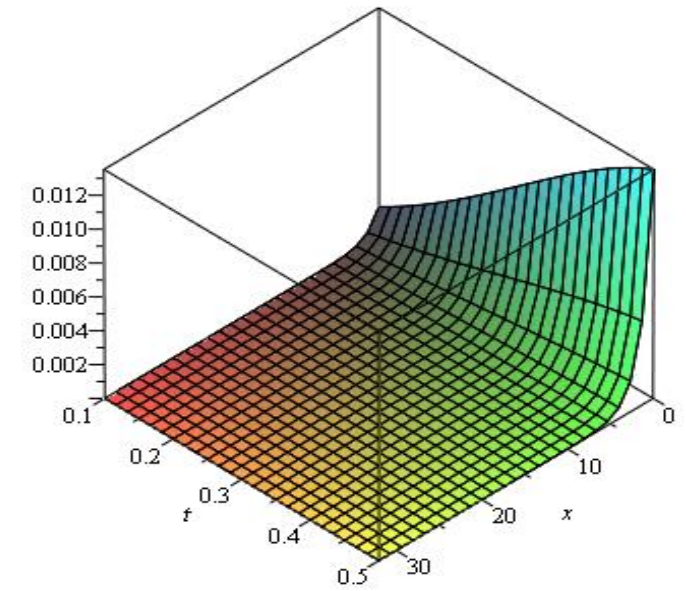

Figure 3. The absolute error for the two borders approximation (Y-2) between NIM and the exact solution when $\mathrm{t}=0.1$

\section{SUGGESTED METHOD:}

The basic idea of the proposal technique is to find the optimal parameters of nonlinear KuramotoSivashinsky equation (kse) using the (PSO) algorithm with the (NIM) method. The algorithm uses a series of (NIM) solutions to model the function of the algorithm using the next equations:

$$
\begin{aligned}
& B(a, b, \lambda)=\sum_{i=1}^{c} \sum_{j=1}^{l}\left(B\left(h_{i}, t_{j}\right)-\hat{B}\left(h_{i}, t_{j}\right)\right)^{2} \\
& F=\frac{1}{2}(Y(\alpha, k, \mathrm{r}))^{2}
\end{aligned}
$$

(c) and (l) represent the gross number, steps utilized in the solution of nonlinear Kuramoto-Sivashinsky equation (KSE). $(c)$ and $(l)$ represent the gross number of steps utilized in the solution domain of and respectively, (B) the solution of nonlinear Kuramoto-Sivashinsky equation (KSE), and $\hat{B}$ is the exact 
solutions for this equation. (F) show the fitness function (mean square error) is solved by utilize the PSO. Consequently, the optimal worths of system (15-16) are obtained through the following:

$$
\begin{aligned}
& \alpha=-1 \\
& k=1 \\
& r=-10
\end{aligned}
$$

The error in the proposed strategy turned out to have reached 10-19 at its lowest level and 10-32

\begin{tabular}{|c|c|c|c|c|c|}
\hline \multicolumn{6}{|c|}{$\mathrm{T}$} \\
\hline $\mathrm{H}$ & 0.1 & 0.2 & 0.3 & 0.4 & 0.5 \\
\hline 8 & $2.383889227 * 10^{-24}$ & $1.886040853 * 10^{-23}$ & $6.34691249 * 10^{-23}$ & $1.503480121 * 10^{-22}$ & $2.937733963 * 10^{-22}$ \\
\hline 15 & 0 & 0 & 0 & 0 & 0 \\
\hline 17 & $4.558541467 * 10^{-21}$ & $3.206550678 * 10^{-20}$ & $1.03630777 * 10^{-19}$ & $2.403642352 * 10^{-19}$ & $4.633757616^{*} 10^{-19}$ \\
\hline 20 & $7.357197295 * 10^{-25}$ & $5.543562616 * 10^{-24}$ & $1.83245542 * 10^{-23}$ & $4.297972002 * 10^{-23}$ & $8.341008569 * 10^{-23}$ \\
\hline 30 & $3.150580238 * 10^{-32}$ & $2.224282936 * 10^{-31}$ & $7.19758023 * 10^{-31}$ & $1.670485542 * 10^{-30}$ & $3.221601400 * 10^{-30}$ \\
\hline
\end{tabular}
at its best, as shown in Table 2 and Figures 4 and 5.

Table 2. Absolute error of the $\left|B_{\text {exact }}-B_{\text {NIM }}\right|$ Using the parameters chosen by pso

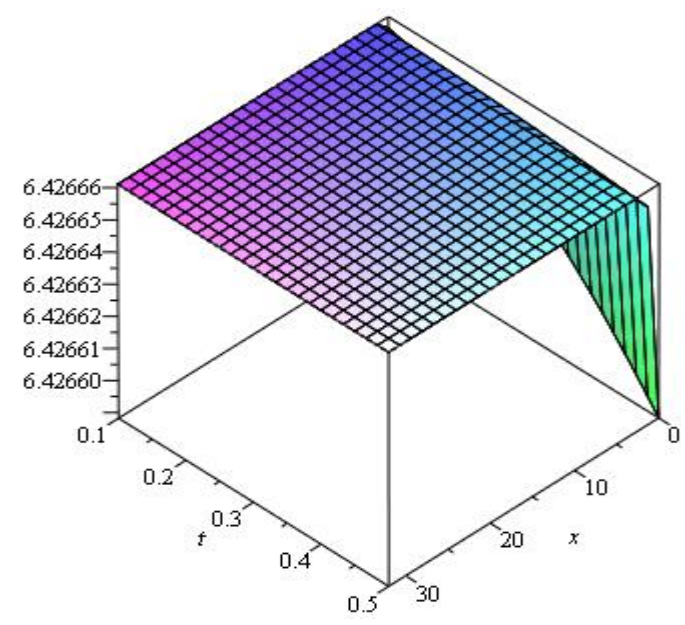

Figure 4. The function $(B)$ for the two borders approximation $(B-2)$ by using NIM with the PSO algorithm, when $\mathrm{t}=0.1$

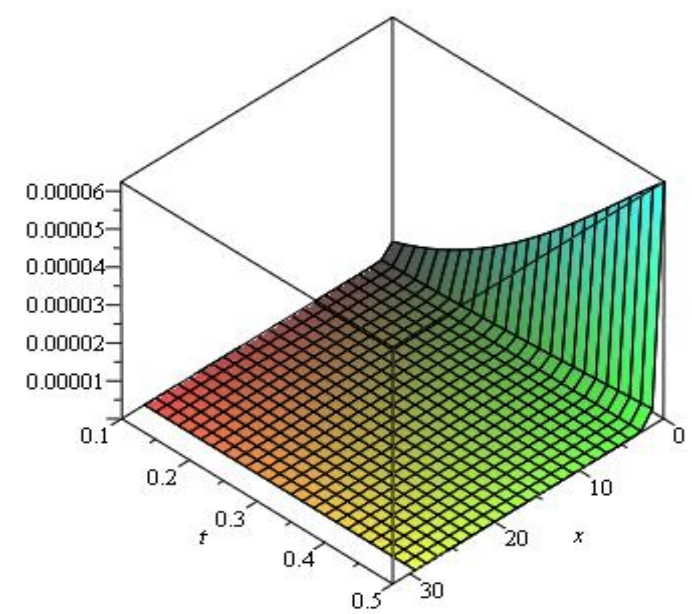

Figure 5. The absolute error for the two borders approximation $(B-2)$ between NIM and the exact solution when $\mathrm{t}=0.1$, with the $(\mathrm{PSO})$ algorithm

\section{CONCLUSION}

In this article, the Kuramoto-Sivashinsky equation was successfully studied and solved in a new iterative method. The results showed great convergence when compared with the exact solution. The results were very clear when using the particle swarm optimization (PSO) algorithm in finding the best parameters, $(\mathrm{k}=1, \mathrm{r}=-10)$, respectively. Which, in turn has had a strong and direct influence in the improvement of the solution to a large degree and obtain an excellent convergence and very efficient when compared to the exact solution.

\section{ACKNOWLEDGMENTS}

The author is very grateful to the University of Mosul /College of Computer Sciences and Mathematics, for their provided facilities, which helped to improve the quality of this work.

\section{REFERENCES}

[1] M. A. A. Alhafedh and O. S. Qasim, "Two-Stage Gene Selection in Microarray Dataset Using Fuzzy Mutual Information and Binary Particle Swarm Optimization, Indian Journal of Forensic Medicine \& Toxicology, vol. 13, pp. 1162-1171, 2019. 
[2] A. S. Al-Obeidi and S. F. Al-Azzawi, "Chaos Synchronization in a 6-D Hyperchaotic System with Self-Excited Attractor," TELKOMNIKA Telecommunication, Computing, Electronics and Control, vol. 18, no 3, June 2020.

[3] A. Hemeda, "New iterative method: application to the nth order integro-differential equations", Information B, vol. 16, no. 6, pp. 3841-3852, 2013.

[4] S. Bhalekar and V. Daftardar-Gejji. "Convergence of the new iterative method", International Journal of Differential Equations, 2011.

[5] A. Hemeda and M. Saleh Alluhaibi. "New iterative method for solving gas dynamic equation", International Journal of Applied Mathematical Research, vol. 3, no. 2, pp. 190-195, 2014.

[6] Podlubny, Fractional Differential Equations, Academic Press, New York, NY, USA, 1999.

[7] K. Al-Khaled and S. Momani, "An approximate solution for a fractional diffusion-wave equation using the decomposition method," Applied Mathematics and Computation, vol. 165, no. 2, pp. 473-483, 2005.

[8] A.Hanyga, "Multidimensional solutions of time-fractional diffusion-wave equations," The Royal Society of London A, vol. 458, no. 2020, pp. 933-957, 2002.

[9] V.Daftardar-Gejji andH. Jafari, "An iterative method for solving nonlinear functional equations," Journal of Mathematical Analysis and Applications, vol. 316, no. 2, pp.753-763, 2006.

[10] A. A. Hemeda, "New iterative method: application to the nthorder integro- differential equations," Information B, vol. 16, no. 6, pp. 3841-3852, 2013.

[11] S. Bhalekar and V. Daftardar-Gejji, "New iterative method: application to partial differential equations," Applied Mathematics and Computation, vol. 203, no. 2, pp. 778-783, 2008.

[12] Manoj Kumar, Anuj Shanker Saxena, "new iterative method for solving higher order Kdv equations",4th international conference on science, technology and management, India international Centre, New Delhi, (ICSTM16), 15 May 2016, Isbn;978-81-932074-8-2.

[13] Dongshu Wang Dapei Tan1 · Lei Liu, "Particle swarm optimization algorithm: an overview", pp:387-408, 2018.

[14] Chong Tian1, Chunshan Xue, Yongfeng Cui,"Application of Partial Differential Equation Based on PSO Algorithm ", ISSN: 1473-804x online, 1473-8031 print.

[15] G. I. Sivashinsky, "Nonlinear analysis of hydrodynamic instability in laminar flames I: Derivation of basic equations", Acta Astronau, vol. 4, pp. 1177-1206, 1977.

[16] Y. Kuramoto, "Diffusion-induced chaos in reaction systems", Suppl. Prog. Theor. Phys, 64, (1978), pp. 346-367.

[17] Z. Sh. Al-Talib, S. F. AL-Azzawi, "Projective and Hybrid Projective Synchronization of 4-D Hyperchaotic System Via Nonlinear Controller Strategy," TELKOMNIKA Telecommunication, Computing, Electronics and Control, vol. 18, no. 2, April 2020.

[18] S. Y. Al-hayali and S. F. AL-Azzawi, "An Optimal Control for Complete Synchronization of 4D Rabinovich Hyperchaotic Systems," TELKOMNIKA Telecommunication, Computing, Electronics and Control vol. 18, no 2, pp. 994-1000, Apr 2020.

[19] S. F. Al-Azzawi and M. M. Aziz, "Chaos Synchronization of Nonlinear Dynamical Systems via a Novel Analytical Approach,” Alexandria Engineering Journal, vol. 57, no. 4, pp. 3493-3500, Dec 2018.

[20] S. F. Al-Azzawi and M. M. Aziz, "Strategies of Linear Feedback Control and its classification," TELKOMNIKA Telecommunication, Computing, Electronics and Control, vol. 17, no. 4, pp. 1931-1940, Aug 2019.

[21] S. Al-hayali, S. F. AL-Azzawi, "An Optimal Nonlinear Control for Anti-Synchronization of Rabinovich Hyperchaotic System," Indonesian Journal of Electrical Engineering and Computer Science, vol. 19, no. 1, July 2020.

[22] Muhammet Kurulay, Aydin Secer and Mehmet Ali Akinlar, "A New Approximate Analytical Solution of Kuramoto -Sivashinsky Equation Using Homotopy Analysis Method", Applied Mathematics \& Information Sciences, no. 1, pp. 267-271 2013.

[23] Z. N. Al-kateeb and M. R. Al-Bazaz, "Steganography in Colored Images Based on Biometrics," Tikrit Journal of Pure Science, vol. 24, no. 3, pp. 111-117, May 2019.

[24] A. S. Al-Obeidi and S. F. Al-Azzawi, "Projective Synchronization for a Class of 6-D Hyperchaotic Lorenz System," Indonesian Journal of Electrical Engineering and Computer Science, vol. 16, no. 2, pp. 692-700, Nov 2019.

[25] Brajesh Kumar Singh, Geeta Arora, Pramod Kumar, "A note on solving the fourth-order Kuramoto-Sivashinsky equation by the compact finite difference scheme", Ain Shams Engineering Journal, pp. 1581-1589, 2018.

[26] Uddin, M., Haq, S., Siraj-ul-Islam, "A mesh-free numerical method for solution of the family of KuramotoSivashinsky equations," Appl. Math. Comput. 212, 2009, 458-469.

[27] K. A. Abed \& A. A. Ahmad, "The Best Parameters Selection Using Pso Algorithm to Solving For Ito System by New Iterative Technique," Indonesian Journal of Electrical Engineering and Computer Science, vol. 18, no. 3, pp. 1638-1645, June 2020.

[28] Akrivis, Georgios, Smyrlis, Yiorgos-sokratis, "Implicit-explicit BDF methods for the Kuramoto-Sivashinsky equation," Appl. Numer. Math, vol. 51, pp. 151-169, 2004.

[29] A.F., Qasim, B.J. Salim, "Application New Iterative Method for Solving Modified Korteweg-Devries (MKdV) System from Three Equations" Journal of Advanced Research in Dynamical and Control Systems, vol. 11, pp.1-7, 2019.

[30] John Wiley and Sons, "partial differential equation", 2007.

[31] Majeed A. Yousif, Fadhil Easif, Saad A. Manaa, "Solving the Kuramoto-Sivashinsky equation via Variational Iteration Method", vol. 3, no. 3, pp. 260-264, 2014. 